\title{
Positive solutions of a singular semipositone boundary value problems for fourth-order coupled difference equations
}

Wenjin Li ${ }^{1}$, Yupeng Fei ${ }^{1^{*}}$, Biaoan Shan ${ }^{1}$ and Yanni Pang ${ }^{2}$

"Correspondence: ypfei@126.com 'School of Management, Jilin University, Changchun, Jilin 130012, P.R. China

Full list of author information is available at the end of the article

$$
\begin{aligned}
& \text { Abstract } \\
& \text { In this article, we study the boundary value problems } \\
& \text { coupled difference equations } \\
& \qquad\left\{\begin{array}{l}
\triangle^{4} u(i-2)=f_{1}(i, v(i))+e_{1}(i), \quad i \in[2, T+2], \\
\triangle^{4} v(i-2)=f_{2}(i, u(i))+e_{2}(i), \quad i \in[2, T+2], \\
u(0)=u(1)=v(1)=v(0)=0, \\
u(T+3)=u(T+4)=v(T+4)=v(T+3)=0 .
\end{array}\right.
\end{aligned}
$$

In this article, we study the boundary value problems for the fourth-order nonlinear

Throughout our nonlinearity may be singular. We establish the existence of positive solutions for the fourth-order coupled systems. The proof relies on Schauder's fixed point theorem.

MSC: 39A11

Keywords: positive solution; positone and semipositone boundary value problem; singular difference equation; fixed point theorem

\section{Introduction}

In this article, we consider the following boundary value problems of difference equations

$$
\left\{\begin{array}{l}
\triangle^{4} u(i-2)=f_{1}(i, v(i))+e_{1}(i), \quad i \in[2, T+2], \\
\triangle^{4} v(i-2)=f_{2}(i, u(i))+e_{2}(i), \quad i \in[2, T+2], \\
u(0)=u(1)=v(1)=v(0)=0, \\
u(T+3)=u(T+4)=v(T+4)=v(T+3)=0,
\end{array}\right.
$$

here $[2, T+2]=\{2,3, \ldots, T+2\}$ and $u, v, e_{j}:[0, T+4] \rightarrow R, f_{j}:[0, T+4] \times R \rightarrow R^{+}, j=1,2$. We will let $[a, b]$ denote the discrete integer set $[a, b]=\{a, a+1, \ldots, b\}, C([a, b])$ denote set of continuous function on $[a, b]$ (discrete topology) with norm $\|\cdot\|=\max _{k \in[a, b]}|\cdot|$.

Recently, many literature on the boundary value of difference equations have appeared. We refer the reader to [1-17] and the references therein, which include work on Agarwal, Eloe, Erber, O’Regan, Henderson, Merdivenci, Yu, Ma et al., concerning the existence of positive solutions and the corresponding eigenvalue problems. Recently, the existence of

2012 Li et al : licensee Springer. This is an Open Access article distributed under the terms of the Creative Commons Attribution License (http://creativecommons.org/licenses/by/2.0), which permits unrestricted use, distribution, and reproduction in any medium, provided the original work is properly cited. 
positive solutions of fourth-order discrete boundary value problems have been studied by several authors, for examples, see [16-18] and the references therein.

The fourth-order boundary value problems of ordinary value problems have important application in various branches of pure and applied science. As in entrepreneurial network evolution studies, the research paradigm of ' $U$ related to $V$ ' was always applied, while $U$ or $\mathrm{V}$ was the measurement of the attitudes of the entrepreneurs who answered the questionnaire. According to cognitive psychology studies $[19,20]$, questionnaire shows the change of attitude, which is the fourth-order dependent of original signal. So whether this paradigm will lead to meaningful causal outcome, which is basically depended on difference equations.

The fourth-order boundary value problems of ordinary value problems have important application in various branches of pure and applied science. They arise in the mathematical modeling of viscoelastic and inelastic flows, deformation of beams and plate deflection theory [21-23]. For examples, the deformations of an elastic beam can be described by the boundary value problems of the fourth-order ordinary differential equations. There have been extensive studies on fourth-order boundary value problems with diverse boundary conditions via many methods, for example [24-27] and the references therein.

The remaining part of the article is organized as follows. In Section "Preliminaries", some preliminary results will be given. In the remaining sections, by employing a basic application of Schauder's fixed point theorem, we state and prove the existence results for (1). Our view point sheds some new light on problems with weak force potentials and prove that in some situations weak singularities may stimulate the existence of positive solutions.

\section{Preliminaries}

In this section, we state the preliminary information that we need to prove the main results. From $[4,5]$, we have the following lemma.

Lemma $1 x(i)$ is a solution of equation

$$
\left\{\begin{array}{l}
\triangle^{4} x(i-2)=h(i), \quad i \in[2, T+2] \\
x(0)=x(1)=x(T+3)=x(T+4)=0
\end{array}\right.
$$

if only and if

$$
x(i)=\sum_{j=2}^{T+2} G(i, j) h(j), \quad i \in[0, T+4],
$$

where

$$
G(i, j)= \begin{cases}\frac{(T+4-i)^{2}(j-1)^{2}}{2}\left(\frac{i}{(T+3)^{2}}-\frac{(T+4+2 i)(j-1)}{3(T+4)^{3}}\right), & 2 \leq j \leq i+1, \\ \frac{i^{2}(T+4-j)^{2}}{6}\left(\frac{(T+3-i)(T+4+2 j)}{(T+4)^{3}}-\frac{T+4-j}{(T+3)^{2}}\right), & i+1<s \leq T+2 .\end{cases}
$$

Lemma 2 The Green's function $G(i, j)$ defined by (4) have properties

$$
\begin{aligned}
& C_{0} i^{2}(T+4-i)^{2}(j-1)^{2}(T+4-j)^{2} \leq G(i, j) \leq(j-1)^{2}(T+4-j)^{2}, \\
& G(i, j) \leq i^{2}(T+4-i)^{2},
\end{aligned}
$$

where $C_{0}=\frac{1}{3(T+4)^{7}}$. 
For our constructions, we shall consider the Banach space $E=C([0, T+4])$ equipped with the standard norm $\|x\|=\max _{0 \leq i \leq T+4}|x(i)|, x \in E$. We define a cone $P \subset E$ by

$$
P=\left\{x \in E \mid x(i) \geq C_{0} i^{2}(T+4-i)^{2}\|x\|, i \in[0, T+4]\right\} .
$$

Define an operator $A(u, v)=\left(A_{1}(u), A_{2}(v)\right): P \times P \rightarrow E \times E$ by

$$
\begin{aligned}
& A_{1} u(i)=\sum_{j=2}^{T+2} G(i, j)\left(f_{1}(i, v(i))+e_{1}(i)\right), \quad i \in[0, T+4], v \in P, \\
& A_{2} v(i)=\sum_{j=2}^{T+2} G(i, j)\left(f_{2}(i, u(i))+e_{2}(i)\right), \quad i \in[0, T+4], u \in P .
\end{aligned}
$$

Notice from (5) and Lemma 2 that, for $u \in P$, we have

$$
\begin{aligned}
& A_{1} u(i)=\sum_{j=2}^{T+2} G(i, j)\left(f_{1}(i, v(i))+e_{1}(i)\right) \leq \sum_{j=2}^{T+2}(j-1)^{2}(T+4-j)^{2}\left(f_{1}(i, v(i))+e_{1}(i)\right), \\
& A_{2} v(i)=\sum_{j=2}^{T+2} G(i, j)\left(f_{2}(i, u(i))+e_{2}(i)\right) \leq \sum_{j=2}^{T+2}(j-1)^{2}(T+4-j)^{2}\left(f_{2}(i, u(i))+e_{2}(i)\right),
\end{aligned}
$$

then $\left\|A_{1} v\right\| \leq \sum_{j=2}^{T+2}(j-1)^{2}(T+4-j)^{2}\left(f_{1}(i, v(i))+e_{1}(i)\right)$ and $\left\|A_{2} u\right\| \leq \sum_{j=2}^{T+2}(j-1)^{2}(T+4-$ $j)^{2}\left(f_{2}(i, u(i))+e_{2}(i)\right)$.

On the other hand, we have

$$
\begin{aligned}
A_{1} u(i) & \geq C_{0} i^{2}(T+4-i)^{2} \sum_{j=2}^{T+2}(j-1)^{2}(T+4-j)^{2}\left(f_{1}(i, v(i))+e_{1}(i)\right) \\
& \geq C_{0} i^{2}(T+4-i)^{2}\left\|A_{1} u\right\|, \\
A_{2} v(i) & \geq C_{0} i^{2}(T+4-i)^{2} \sum_{j=2}^{T+2}(j-1)^{2}(T+4-j)^{2}\left(f_{2}(i, u(i))+e_{2}(i)\right) \\
& \geq C_{0} i^{2}(T+4-i)^{2}\left\|A_{2} v\right\| .
\end{aligned}
$$

Thus, $A(P \times P) \subset P \times P$. In addition, standard arguments show that $A$ is completely continuous.

Now, we define the function $\gamma_{k}: E \rightarrow E$ by

$$
\gamma_{k}(i)=\sum_{j=2}^{T+2} \frac{G(i, j)}{i^{2}(T+4-i)^{2}} e_{k}(j), \quad i \in[0, T+4], k=1,2,
$$

which is the unique solution of

$$
\left\{\begin{array}{l}
\triangle^{4} x(i-2)=e_{k}(i), \quad i \in[2, T+2], \\
x(0)=x(1)=x(T+3)=x(T+4)=0 .
\end{array}\right.
$$


Throughout this article, we use the following notations

$$
\gamma_{k^{*}}=\min _{k, i} \gamma_{k}(i), \quad \gamma_{k}^{*}=\max _{k, i} \gamma_{k}(i)
$$

\section{The case $\gamma_{1^{*}} \geq 0, \gamma_{2^{*}} \geq 0$}

Theorem 1 Assume that there exists $b_{k} \succ 0, \hat{b}_{k} \succ 0$ and $0<\alpha_{k}<1$ such that

$$
0 \leq \frac{\hat{b}_{k}(i)}{x^{\alpha_{k}}} \leq f_{k}(i, x) \leq \frac{b_{k}(i)}{x^{\alpha_{k}}}, \quad \text { for all } x>0, i \in[2, T+2], k=1,2
$$

If $\gamma_{1^{*}} \geq 0, \gamma_{2^{*}} \geq 0$, then there exists a positive solution of (1).

Proof A solution of (1) is just a fixed point of the completely continuous map $A(x, y)=$ $\left(A_{1} x, A_{2} y\right): P \times P \rightarrow P \times P$, from (5) we have

$$
\begin{aligned}
& \left(A_{1} u\right)(t)=\sum_{j=2}^{T+2} G(i, j) f_{1}(i, v(i))+\gamma_{1}(i), \\
& \left(A_{2} v\right)(t)=\sum_{j=2}^{T+2} G(i, j) f_{2}(i, u(i))+\gamma_{2}(i) .
\end{aligned}
$$

By a direct application of Schauder's fixed point theorem, the proof is finished if we prove that $A$ maps the closed convex set defined as

$$
\begin{aligned}
K= & \left\{(u, v) \in P \times P: r_{1} i^{2}(T+4-i)^{2} \leq u(i) \leq R_{1} i^{2}(T+4-i)^{2},\right. \\
& \left.r_{2} i^{2}(T+4-i)^{2} \leq v(i) \leq R_{2} i^{2}(T+4-i)^{2}, i \in[0, T+4]\right\},
\end{aligned}
$$

into itself, where $R_{1}>r_{1}>0, R_{2}>r_{2}>0$ are positive constants to be fixed properly. For convenience, we introduce the following notations

$$
\beta_{k}(i)=\sum_{j=2}^{T+2} \frac{G(i, j)}{i^{2}(T+4-i)^{2}} b_{k}(j), \quad \hat{\beta}_{k}(i)=\sum_{j=2}^{T+2} \frac{G(i, j)}{i^{2}(T+4-i)^{2}} \hat{b}_{k}(j), \quad k=1,2 .
$$

Given $(u, v) \in K$, by the nonnegative sign of $G$ and $f_{k}, k=1,2$, we have

$$
\begin{aligned}
\left(A_{1} u\right)(i) & =i^{2}(T+4-i)^{2} \sum_{j=2}^{T+2} \frac{G(i, j)}{i^{2}(T+4-i)^{2}} f_{1}(j, v(j))+\gamma_{1}(i) \\
& \geq i^{2}(T+4-i)^{2} \sum_{j=2}^{T+2} \frac{G(i, j)}{i^{2}(T+4-i)^{2}} \frac{\hat{b}_{1}(j)}{v^{\alpha_{1}}(j)}+i^{2}(T+4-i)^{2} \gamma_{1^{*}} \\
& \geq i^{2}(T+4-i)^{2} \sum_{j=2}^{T+2} \frac{G(i, j)}{i^{2}(T+4-i)^{2}} \frac{\hat{b}_{1}(j)}{R_{2}^{\alpha_{1}}} \\
& \geq i^{2}(T+4-i)^{2} \hat{\beta}_{1^{*}} \cdot \frac{1}{R_{2}^{\alpha_{1}}} .
\end{aligned}
$$


Note for every $(u, v) \in K$

$$
\begin{aligned}
\left(A_{1} u\right)(i) & =i^{2}(T+4-i)^{2} \sum_{j=2}^{T+2} \frac{G(i, j)}{i^{2}(T+4-i)^{2}} f_{1}(j, v(j))+\gamma_{1}(i) \\
& \leq i^{2}(T+4-i)^{2} \sum_{j=2}^{T+2} \frac{G(i, j)}{i^{2}(T+4-i)^{2}} \frac{b_{1}(j)}{v^{\alpha_{1}}(j)}+i^{2}(T+4-i)^{2} \gamma_{1}^{*} \\
& \leq i^{2}(T+4-i)^{2} \sum_{j=2}^{T+2} \frac{G(i, j)}{i^{2}(T+4-i)^{2}} \frac{b_{1}(j)}{r_{2}^{\alpha_{1}}}+i^{2}(T+4-i)^{2} \gamma_{1}^{*} \\
& \leq i^{2}(T+4-i)^{2}\left(\beta_{1}^{*} \cdot \frac{1}{\left.r_{2}^{\alpha_{1}}+\gamma_{1}^{*}\right) .}\right.
\end{aligned}
$$

Similarly, by the same strategy, we have

$$
\begin{aligned}
\left(A_{2} v\right)(i) & =i^{2}(T+4-i)^{2} \sum_{j=2}^{T+2} \frac{G(i, j)}{i^{2}(T+4-i)^{2}} f_{2}(j, u(j))+\gamma_{2}(i) \\
& \geq i^{2}(T+4-i)^{2} \sum_{j=2}^{T+2} \frac{G(i, j)}{i^{2}(T+4-i)^{2}} \frac{\hat{b}_{2}(j)}{u^{\alpha_{2}}(j)}+i^{2}(T+4-i)^{2} \gamma_{2^{*}} \\
& \geq i^{2}(T+4-i)^{2} \sum_{j=2}^{T+2} \frac{G(i, j)}{i^{2}(T+4-i)^{2}} \frac{\hat{b}_{2}(j)}{R_{1}^{\alpha_{2}}} \\
& \geq i^{2}(T+4-i)^{2} \hat{\beta}_{2^{*}} \cdot \frac{1}{R_{1}^{\alpha_{2}}}, \\
\left(A_{2} v\right)(i) & =i^{2}(T+4-i)^{2} \sum_{j=2}^{T+2} \frac{G(i, j)}{i^{2}(T+4-i)^{2}} f_{2}(j, u(j))+\gamma_{2}(i) \\
& \leq i^{2}(T+4-i)^{2} \sum_{j=2}^{T+2} \frac{G(i, j)}{i^{2}(T+4-i)^{2}} \frac{b_{2}(j)}{u^{\alpha_{2}}(j)}+i^{2}(T+4-i)^{2} \gamma_{2}^{*} \\
& \leq i^{2}(T+4-i)^{2} \sum_{j=2}^{T+2} \frac{G(i, j)}{i^{2}(T+4-i)^{2}} \frac{b_{2}(j)}{r_{1}^{\alpha_{2}}}+i^{2}(T+4-i)^{2} \gamma_{2}^{*} \\
& \leq i^{2}(T+4-i)^{2}\left(\beta_{2}^{*} \cdot \frac{1}{\left.r_{1}^{\alpha_{2}}+\gamma_{2}^{*}\right) .}\right.
\end{aligned}
$$

Thus $\left(A_{1} u, A_{2} v\right) \in K$ if $r_{1}, r_{2}, R_{1}$ and $R_{2}$ are chosen so that

$$
\begin{array}{ll}
\hat{\beta}_{1^{*}} \cdot \frac{1}{R_{2}^{\alpha_{1}}} \geq r_{1}, & \beta_{1}^{*} \cdot \frac{1}{r_{2}^{\alpha_{1}}}+\gamma_{1}^{*} \leq R_{1}, \\
\hat{\beta}_{2^{*}} \cdot \frac{1}{R_{1}^{\alpha_{2}}} \geq r_{2}, & \beta_{2}^{*} \cdot \frac{1}{r_{1}^{\alpha_{2}}}+\gamma_{2}^{*} \leq R_{2} .
\end{array}
$$

Note that $\hat{\beta}_{i^{*}}, \beta_{i^{*}}>0$ and taking $R=R_{1}=R_{2}, r=r_{1}=r_{2}, r=\frac{1}{R}$, it is sufficient to find $R>1$ such that

$$
\hat{\beta}_{1^{*}} \cdot R^{1-\alpha_{1}} \geq 1, \quad \beta_{1}^{*} \cdot R^{\alpha_{1}}+\gamma_{1}^{*} \leq R
$$




$$
\hat{\beta}_{2^{*}} \cdot R^{1-\alpha_{2}} \geq 1, \quad \beta_{2}^{*} \cdot R^{\alpha_{2}}+\gamma_{2}^{*} \leq R
$$

and these inequalities hold for $R$ big enough because $\alpha_{i}<1$. The proof is complete.

The case $\gamma_{1}^{*} \leq 0, \gamma_{2}^{*} \leq 0$

The aim of this section is to show that the presence of a weak singular nonlinearity makes it possible to find positive solutions if $\gamma_{1}^{*} \leq 0, \gamma_{2}^{*} \leq 0$.

Theorem 2 Assume that there exist $b_{k}, \hat{b}_{k} \succ 0$ and $0<\alpha_{k}<1$, such that $(H)$ is satisfied. If $\gamma_{1}^{*} \leq 0, \gamma_{2}^{*} \leq 0$, and

$$
\begin{aligned}
& \gamma_{1^{*}} \geq\left[\alpha_{1} \alpha_{2} \cdot \frac{\hat{\beta}_{1^{*}}}{\left(\beta_{2}^{*}\right)^{\alpha_{1}}}\right]^{\frac{1}{1-\alpha_{1} \alpha_{2}}}\left(1-\frac{1}{\alpha_{1} \alpha_{2}}\right), \\
& \gamma_{2^{*}} \geq\left[\alpha_{1} \alpha_{2} \cdot \frac{\hat{\beta}_{2^{*}}}{\left(\beta_{1}^{*}\right)^{\alpha_{2}}}\right]^{\frac{1}{1-\alpha_{1} \alpha_{2}}}\left(1-\frac{1}{\alpha_{1} \alpha_{2}}\right),
\end{aligned}
$$

then there exists a positive solution of (1).

Proof We follow the same strategy and notation as in the proof of ahead theorem. In this case, to prove that $A: K \rightarrow K$, it is sufficient to find $0<r_{1}<R_{1}, 0<r_{2}<R_{2}$ such that

$$
\begin{array}{ll}
\frac{\hat{\beta}_{1^{*}}}{R_{2}^{\alpha_{1}}}+\gamma_{1^{*}} \geq r_{1}, & \frac{\beta_{1}^{*}}{r_{2}^{\alpha_{1}}} \leq R_{1}, \\
\frac{\hat{\beta}_{2^{*}}}{R_{1}^{\alpha_{2}}}+\gamma_{2^{*}} \geq r_{2}, & \frac{\beta_{2}^{*}}{r_{1}^{\alpha_{2}}} \leq R_{2} .
\end{array}
$$

If we fix $R_{1}=\frac{\beta_{1}^{*}}{r_{2}^{\alpha_{1}}}, R_{2}=\frac{\beta_{2}^{*}}{r_{1}^{\alpha_{2}}}$, then the first inequality of (8) holds if $r_{2}$ satisfies

$$
\hat{\beta}_{2^{*}}\left(\beta_{1}^{\prime \prime}\right)^{-\alpha_{2}} r_{2}^{\alpha_{1} \alpha_{2}}+\gamma_{2^{*}} \geq r_{2}
$$

or equivalently

$$
\gamma_{2^{*}} \geq g\left(r_{2}\right):=r_{2}-\frac{\hat{\beta}_{2^{*}}}{\left(\beta_{1}^{*}\right)^{\alpha_{2}}} r_{2}^{\alpha_{1} \alpha_{2}}
$$

The function $g\left(r_{2}\right)$ possesses a minimum at

$$
r_{20}:=\left[\alpha_{1} \alpha_{2} \cdot \frac{\hat{\beta}_{2^{*}}}{\left(\beta_{1}^{*}\right)^{\alpha_{2}}}\right]^{\frac{1}{1-\alpha_{1} \alpha_{2}}}
$$

Taking $r_{2}=r_{20}$, then (6) holds if

$$
\gamma_{2^{*}} \geq g\left(r_{20}\right)=\left[\alpha_{1} \alpha_{2} \cdot \frac{\hat{\beta}_{2^{*}}}{\left(\beta_{1}^{*}\right)^{\alpha_{2}}}\right]^{\frac{1}{1-\alpha_{1} \alpha_{2}}}\left(1-\frac{1}{\alpha_{1} \alpha_{2}}\right) .
$$

Similarly,

$$
\gamma_{1^{*}} \geq h\left(r_{1}\right):=r_{1}-\frac{\hat{\beta}_{1^{*}}}{\left(\beta_{2}^{*}\right)^{\alpha_{1}}} r_{1}^{\alpha_{1} \alpha_{2}}
$$


$h\left(r_{1}\right)$ possesses a minimum at

$$
\begin{aligned}
& r_{10}:=\left[\alpha_{1} \alpha_{2} \cdot \frac{\hat{\beta}_{1^{*}}}{\left(\beta_{2}^{*}\right)^{\alpha_{1}}}\right]^{\frac{1}{1-\alpha_{1} \alpha_{2}}}, \\
& \gamma_{1^{*}} \geq\left[\alpha_{1} \alpha_{2} \cdot \frac{\hat{\beta}_{1^{*}}}{\left(\beta_{2}^{*}\right)^{\alpha_{1}}}\right]^{\frac{1}{1-\alpha_{1} \alpha_{2}}}\left(1-\frac{1}{\alpha_{1} \alpha_{2}}\right) .
\end{aligned}
$$

Taking $r_{1}=r_{10}, r_{2}=r_{20}$, then the first inequalities in (7) and (8) hold if $\gamma_{1^{*}} \geq g\left(r_{1}\right)$ and $\gamma_{2^{*}} \geq g\left(r_{2}\right)$, which are just condition (6). The second inequalities hold directly from the choice of $R_{1}$ and $R_{2}$, so it remains to prove that $R_{1}=\frac{\beta_{1}^{*}}{r_{20}}>r_{10}, R_{2}=\frac{\beta_{2}^{*}}{r_{10}}>r_{20}$. This is easily verified through elementary computations:

$$
\begin{aligned}
& R_{1}=\frac{\beta_{1}^{*}}{r_{20}^{\alpha_{1}}}=\frac{\beta_{1}^{*}}{\left\{\left[\alpha_{1} \alpha_{2} \cdot \frac{\hat{\beta}_{2^{*}}}{\left(\beta_{1}^{*}\right)^{\alpha_{2}}}\right]^{\frac{1}{1-\alpha_{1} \alpha_{2}}}\right\}^{\alpha_{1}}} \\
& =\frac{\beta_{1}^{*}}{\left[\alpha_{1} \alpha_{2} \cdot \frac{\hat{\beta}_{2^{*}}}{\left(\beta_{1}^{*}\right)^{\alpha_{2}}}\right]^{\frac{\alpha_{1}}{1-\alpha_{1} \alpha_{2}}}}=\frac{\left(\beta_{1}^{*}\right)^{1+\frac{\alpha_{1} \alpha_{2}}{1-\alpha_{1} \alpha_{2}}}}{\left(\alpha_{1} \alpha_{2} \cdot \hat{\beta}_{2^{*}}\right)^{\frac{\alpha_{1}}{1-\alpha_{1} \alpha_{2}}}} \\
& =\frac{\left(\beta_{1}^{*}\right)^{\frac{1}{1-\alpha_{1} \alpha_{2}}}}{\left[\left(\alpha_{1} \alpha_{2} \cdot \hat{\beta}_{2^{*}}\right)^{\alpha_{1}}\right]^{\frac{1}{1-\alpha_{1} \alpha_{2}}}}=\left[\frac{\beta_{1}^{*}}{\left(\alpha_{1} \alpha_{2} \cdot \hat{\beta}_{2^{*}}\right)^{\alpha_{1}}}\right]^{\frac{1}{1-\alpha_{1} \alpha_{2}}} \\
& =\left[\frac{1}{\left(\alpha_{1} \alpha_{2}\right)^{\alpha_{1}}} \cdot \frac{\beta_{1}^{*}}{\left(\hat{\beta}_{2^{*}}\right)^{\alpha_{1}}}\right]^{\frac{1}{1-\alpha_{1} \alpha_{2}}}>\left[\alpha_{1} \alpha_{2} \cdot \frac{\hat{\beta}_{1^{*}}}{\left(\beta_{2}^{*}\right)^{\alpha_{1}}}\right]^{\frac{1}{1-\alpha_{1} \alpha_{2}}}=r_{10} \text {, }
\end{aligned}
$$

since $\hat{\beta}_{k^{*}} \leq \beta_{k}^{*}, k=1,2$. Similarly, we have $R_{2}>r_{20}$. The proof is complete.

The case $\gamma_{1^{*}} \geq 0, \gamma_{2}^{*} \leq 0\left(\gamma_{1}^{*} \leq 0, \gamma_{2^{*}} \geq 0\right)$

Theorem 3 Assume $(H)$ is satisfied. If $\gamma_{1^{*}} \geq 0, \gamma_{2}^{*} \leq 0$ and

$$
\gamma_{2^{*}} \geq r_{21}-\hat{\beta}_{2^{*}} \cdot \frac{r_{21}^{\alpha_{1} \alpha_{2}}}{\left(\beta_{1}^{*}+\gamma_{1}^{*} r_{21}^{\alpha_{1}}\right)^{\alpha_{2}}}
$$

where $0<r_{21}<+\infty$ is a unique positive solution of the equation

$$
r_{2}^{1-\alpha_{1} \alpha_{2}}\left(\beta_{1}^{*}+\gamma_{1}^{*} \cdot r_{2}^{\alpha_{1}}\right)^{1+\alpha_{2}}=\alpha_{1} \alpha_{2} \beta_{1}^{*} \hat{\beta}_{2^{*}}
$$

then there exists a positive solution of (1).

Proof In this case,to prove that $A: K \rightarrow K$, it is sufficient to find $r_{1}<R_{1}, r_{2}<R_{2}$ such that

$$
\begin{aligned}
& \frac{\hat{\beta}_{1^{*}}}{R_{2}^{\alpha_{1}}} \geq r_{1}, \quad \frac{\beta_{2}^{*}}{r_{1}^{\alpha_{2}}} \leq R_{2} . \\
& \frac{\hat{\beta}_{2^{*}}}{R_{1}^{\alpha_{2}}}+\gamma_{2^{*}} \geq r_{2}, \quad \frac{\beta_{1}^{*}}{r_{2}^{\alpha_{1}}}+\gamma_{1}^{*} \leq R_{1} .
\end{aligned}
$$


If we fix $R_{2}=\frac{\beta_{2}^{*}}{r_{1}^{2}}$, then the first inequality of (10) holds if $r_{1}$ satisfies

$$
\frac{\hat{\beta}_{1^{*}}}{\left(\beta_{2}^{*}\right)^{\alpha_{1}}} \cdot r_{1}^{\alpha_{1} \alpha_{2}} \geq r_{1}
$$

or equivalently

$$
0<r_{1} \leq\left[\frac{\hat{\beta}_{1^{*}}}{\left(\beta_{2}^{*}\right)^{\alpha_{1}}}\right]^{\frac{1}{1-\alpha_{1} \alpha_{2}}} .
$$

If we chose $r_{1}>0$ small enough, then (12) holds, and $R_{2}$ is big enough.

If we fix $R_{1}=\frac{\beta_{1}^{*}}{r_{2}^{\alpha_{1}}}+\gamma_{1}^{\prime \prime}$ then the first inequality of (11) holds if $r_{2}$ satisfies

$$
\begin{aligned}
\gamma_{2^{*}} & \geq r_{2}-\frac{\hat{\beta}_{2^{*}}}{R_{1}^{\alpha_{2}}} \\
& =r_{2}-\hat{\beta}_{2^{*}} \cdot \frac{1}{\left(\frac{\beta_{1}^{*}}{\left.r_{2}^{\alpha_{1}}+\gamma_{1}^{*}\right)^{\alpha_{2}}}\right.} \\
& =r_{2}-\hat{\beta}_{2^{*}} \cdot \frac{1}{\left(\frac{\beta_{1}^{*}+\gamma_{1}^{*} \cdot r_{2}^{\alpha_{1}}}{r_{2}^{\alpha_{1}}}\right)^{\alpha_{2}}} \\
& =r_{2}-\hat{\beta}_{2^{*}} \cdot \frac{r_{2}^{\alpha_{1} \alpha_{2}}}{\left(\beta_{1}^{*}+\gamma_{1}^{*} \cdot r_{2}^{\alpha_{1}}\right)^{\alpha_{2}}},
\end{aligned}
$$

or equivalently

$$
\gamma_{2^{*}} \geq f\left(r_{2}\right):=r_{2}-\hat{\beta}_{2^{*}} \cdot \frac{r_{2}^{\alpha_{1} \alpha_{2}}}{\left(\beta_{1}^{*}+\gamma_{1}^{*} \cdot r_{2}^{\alpha_{1}}\right)^{\alpha_{2}}} .
$$

According to

$$
\begin{aligned}
f^{\prime}\left(r_{2}\right)= & 1-\hat{\beta}_{2^{*}} \cdot \frac{1}{\left(\beta_{1}^{*}+\gamma_{1}^{*} \cdot r_{2}^{\alpha_{1}}\right)^{2 \alpha_{2}}} \cdot\left[\alpha_{1} \alpha_{2} r_{2}^{\alpha_{1} \alpha_{2}-1}\left(\beta_{1}^{*}+\gamma_{1}^{*} \cdot r_{2}^{\alpha_{1}}\right)^{\alpha_{2}}\right. \\
& \left.-r_{2}^{\alpha_{1} \alpha_{2}} \alpha_{2}\left(\beta_{1}^{*}+\gamma_{1}^{*} \cdot r_{2}^{\alpha_{1}}\right)^{\alpha_{2}-1} \alpha_{1} \gamma_{1}^{*} r_{2}^{\alpha_{1}-1}\right] \\
= & 1-\frac{\hat{\beta}_{2^{*}} \alpha_{1} \alpha_{2} r_{2}^{\alpha_{1} \alpha_{2}-1}}{\left(\beta_{1}^{*}+\gamma_{1}^{*} \cdot r_{2}^{\alpha_{1}}\right)^{\alpha_{2}}}\left[1-\frac{r_{2}^{\alpha_{1}} \gamma_{1}^{*}}{\beta_{1}^{*}+\gamma_{1}^{*} \cdot r_{2}^{\alpha_{1}}}\right] \\
= & 1-\alpha_{1} \alpha_{2} \beta_{1}^{*} \hat{\beta}_{2^{*}} r_{2}^{\alpha_{1} \alpha_{2}-1}\left(\beta_{1}^{*}+\gamma_{1}^{*} \cdot r_{2}^{\alpha_{1}}\right)^{-1-\alpha_{2}}
\end{aligned}
$$

we have $f^{\prime}(0)=-\infty, f^{\prime}(+\infty)=1$, then there exists $r_{21}$ such that $f^{\prime}\left(r_{21}\right)=0$, and

$$
\begin{aligned}
f^{\prime \prime}\left(r_{2}\right)= & -\left[\alpha_{1} \alpha_{2} \beta_{1}^{*} \hat{\beta}_{2^{*}}\left(\alpha_{1} \alpha_{2}-1\right) r_{2}^{\alpha_{1} \alpha_{2}-2}\left(\beta_{1}^{*}+\gamma_{1}^{*} \cdot r_{2}^{\alpha_{1}}\right)^{-1-\alpha_{2}}\right. \\
& \left.+\alpha_{1} \alpha_{2} \beta_{1}^{*} \hat{\beta}_{2^{*}} r_{2}^{\alpha_{1} \alpha_{2}-1}\left(-1-\alpha_{2}\right)\left(\beta_{1}^{*}+\gamma_{1}^{*} \cdot r_{2}^{\alpha_{1}}\right)^{-2-\alpha_{2}} \gamma_{1}^{*} \alpha_{1} r_{2}^{\alpha_{1}-1}\right]>0 .
\end{aligned}
$$

Then the function $f\left(r_{2}\right)$ possesses a minimum at $r_{21}$, i.e., $f\left(r_{21}\right)=\min _{r_{2} \epsilon(0,+\infty)} f\left(r_{2}\right)$.

Note $f^{\prime}\left(r_{21}\right)=0$, then we have

$$
1-\alpha_{1} \alpha_{2} \beta_{1}^{*} \hat{\beta}_{2^{*}} r_{21}^{\alpha_{1} \alpha_{2}-1}\left(\beta_{1}^{*}+\gamma_{1}^{*} \cdot r_{21}^{\alpha_{1}}\right)^{-1-\alpha_{2}}=0,
$$


or equivalently,

$$
r_{21}^{1-\alpha_{1} \alpha_{2}}\left(\beta_{1}^{*}+\gamma_{1}^{*} \cdot r_{21}^{\alpha_{1}}\right)^{1+\alpha_{2}}=\alpha_{1} \alpha_{2} \beta_{1}^{*} \hat{\beta}_{2^{*}} .
$$

Taking $r_{2}=r_{21}$, then the first inequality in (11) holds if $\gamma_{2^{*}} \geq f\left(r_{21}\right)$, which is just condition (9). The second inequality holds directly by the choice of $R_{2}$, and it would remain to prove that $r_{21}<R_{2}$ and $r_{10}<R_{1}$. These inequalities hold for $R_{2}$ big enough and $r_{1}$ small enough. The proof is complete.

Similarly, we have the following theorem.

Theorem 4 Assume (H) are satisfied. If $\gamma_{1}^{\prime \prime} \leq 0, \gamma_{2^{*}} \geq 0$ and

$$
\gamma_{1^{*}} \geq r_{11}-\hat{\beta}_{1^{*}} \cdot \frac{r_{11}^{\alpha_{1} \alpha_{2}}}{\left(\beta_{2}^{*}+\gamma_{2}^{*} r_{11}^{\alpha_{2}}\right)^{\alpha_{1}}},
$$

where $0<r_{11}<+\infty$ is a unique positive solution of the equation

$$
r_{1}^{1-\alpha_{1} \alpha_{2}}\left(\beta_{2}^{*}+\gamma_{2}^{*} \cdot r_{1}^{\alpha_{2}}\right)^{1+\alpha_{1}}=\alpha_{1} \alpha_{2} \beta_{2}^{*} \hat{\beta}_{1^{*}},
$$

then there exists a positive solution of (1).

The case $\gamma_{1^{*}}<0<\gamma_{1}^{*}, \gamma_{2^{*}}<0<\gamma_{2}^{*}$

Theorem 5 Assume $(H)$ is satisfied. If $\gamma_{1^{*}}<0<\gamma_{1}^{*}, \gamma_{2^{*}}<0<\gamma_{2}^{*}$ and

$$
\begin{aligned}
& \gamma_{1^{*}} \geq r_{10}-\hat{\beta}_{1^{*}} \cdot \frac{r_{10}^{\alpha_{1} \alpha_{2}}}{\left(\beta_{2}^{*}+\gamma_{2}^{*} r_{10}^{\alpha_{2}}\right)^{\alpha_{1}}}, \\
& \gamma_{2^{*}} \geq r_{20}-\hat{\beta}_{2^{*}} \cdot \frac{r_{20}^{\alpha_{1} \alpha_{2}}}{\left(\beta_{1}^{*}+\gamma_{1}^{*} r_{20}^{\alpha_{1}}\right)^{\alpha_{2}}},
\end{aligned}
$$

where $0<r_{10}<+\infty$ is a unique positive solution of the equation

$$
r_{1}^{1-\alpha_{1} \alpha_{2}}\left(\beta_{2}^{*}+\gamma_{2}^{*} \cdot r_{1}^{\alpha_{2}}\right)^{1+\alpha_{1}}=\alpha_{1} \alpha_{2} \beta_{2}^{*} \hat{\beta}_{1^{*}},
$$

and $0<r_{20}<+\infty$ is a unique positive solution of the equation

$$
r_{2}^{1-\alpha_{1} \alpha_{2}}\left(\beta_{1}^{*}+\gamma_{1}^{*} \cdot r_{2}^{\alpha_{1}}\right)^{1+\alpha_{2}}=\alpha_{1} \alpha_{2} \beta_{1}^{*} \hat{\beta}_{2^{*}},
$$

then there exists a positive solution of (1).

Proof We follow the same strategy and notation as in the proof of ahead theorem. In this case, to prove that $A: K \rightarrow K$, it is sufficient to find $r_{1}<R_{1}, r_{2}<R_{2}$ such that

$$
\begin{array}{ll}
\frac{\hat{\beta}_{1^{*}}}{R_{2}^{\alpha_{1}}}+\gamma_{1^{*}} \geq r_{1}, & \frac{\beta_{1}^{*}}{r_{2}^{\alpha_{1}}}+\gamma_{1}^{*} \leq R_{1}, \\
\frac{\hat{\beta}_{2^{*}}}{R_{1}^{\alpha_{2}}}+\gamma_{2^{*}} \geq r_{2}, & \frac{\beta_{2}^{*}}{r_{1}^{\alpha_{2}}}+\gamma_{2}^{*} \leq R_{2} .
\end{array}
$$


If we fix $R_{1}=\frac{\beta_{1}^{*}}{r_{2}^{\alpha_{1}}}+\gamma_{1}^{*}, R_{2}=\frac{\beta_{2}^{*}}{r_{1}^{\alpha_{2}}}+\gamma_{2}^{*}$, then the first inequality of (15) holds if $r_{2}$ satisfies

$$
\gamma_{2^{*}} \geq g\left(r_{2}\right):=r_{2}-\hat{\beta}_{2^{*}} \cdot \frac{r_{2}^{\alpha_{1} \alpha_{2}}}{\left(\beta_{1}^{*}+\gamma_{1}^{* \prime} \cdot r_{2}^{\alpha_{1}}\right)^{\alpha_{2}}} .
$$

According to

$$
\begin{aligned}
g^{\prime}\left(r_{2}\right)= & 1-\hat{\beta}_{2^{*}} \cdot \frac{1}{\left(\beta_{1}^{*}+\gamma_{1}^{*} \cdot r_{2}^{\alpha_{1}}\right)^{2 \alpha_{2}}} \cdot\left[\alpha_{1} \alpha_{2} r_{2}^{\alpha_{1} \alpha_{2}-1}\left(\beta_{1}^{*}+\gamma_{1}^{*} \cdot r_{2}^{\alpha_{1}}\right)^{\alpha_{2}}\right. \\
& \left.-r_{2}^{\alpha_{1} \alpha_{2}} \alpha_{2}\left(\beta_{1}^{*}+\gamma_{1}^{*} \cdot r_{2}^{\alpha_{1}}\right)^{\alpha_{2}-1} \alpha_{1} \gamma_{1}^{*} r_{2}^{\alpha_{1}-1}\right] \\
= & 1-\frac{\hat{\beta}_{2^{*}} \alpha_{1} \alpha_{2} r_{2}^{\alpha_{1} \alpha_{2}-1}}{\left(\beta_{1}^{*}+\gamma_{1}^{*} \cdot r_{2}^{\alpha_{1}}\right)^{\alpha_{2}}}\left[1-\frac{r_{2}^{\alpha_{1}} \gamma_{1}^{*}}{\beta_{1}^{*}+\gamma_{1}^{*} \cdot r_{2}^{\alpha_{1}}}\right] \\
= & 1-\alpha_{1} \alpha_{2} \beta_{1}^{*} \hat{\beta}_{2^{*}} r_{2}^{\alpha_{1} \alpha_{2}-1}\left(\beta_{1}^{*}+\gamma_{1}^{*} \cdot r_{2}^{\alpha_{1}}\right)^{-1-\alpha_{2}},
\end{aligned}
$$

we have $g^{\prime}(0)=-\infty, g^{\prime}(+\infty)=1$, then there exists $r_{20}$ such that $g^{\prime}\left(r_{20}\right)=0$, and

$$
\begin{aligned}
g^{\prime \prime}\left(r_{2}\right)= & -\left[\alpha_{1} \alpha_{2} \beta_{1}^{*} \hat{\beta}_{2^{*}}\left(\alpha_{1} \alpha_{2}-1\right) r_{2}^{\alpha_{1} \alpha_{2}-2}\left(\beta_{1}^{*}+\gamma_{1}^{* \prime} \cdot r_{2}^{\alpha_{1}}\right)^{-1-\alpha_{2}}\right. \\
& \left.+\alpha_{1} \alpha_{2} \beta_{1}^{\prime \prime} \hat{\beta}_{2^{* \prime}} r_{2}^{\alpha_{1} \alpha_{2}-1}\left(-1-\alpha_{2}\right)\left(\beta_{1}^{* \prime}+\gamma_{1}^{\prime \prime} \cdot r_{2}^{\alpha_{1}}\right)^{-2-\alpha_{2}} \gamma_{1}^{\prime \prime} \alpha_{1} r_{2}^{\alpha_{1}-1}\right]>0 .
\end{aligned}
$$

Then the function $g\left(r_{2}\right)$ possesses a minimum at $r_{20}$, i.e., $g\left(r_{20}\right)=\min _{r_{2} \epsilon(0,+\infty)} g\left(r_{2}\right)$.

Note $g^{\prime}\left(r_{20}\right)=0$, then we have

$$
1-\alpha_{1} \alpha_{2} \beta_{1}^{*} \hat{\beta}_{2^{*}} r_{21}^{\alpha_{1} \alpha_{2}-1}\left(\beta_{1}^{*}+\gamma_{1}^{*} \cdot r_{21}^{\alpha_{1}}\right)^{-1-\alpha_{2}}=0
$$

or equivalently,

$$
r_{20}^{1-\alpha_{1} \alpha_{2}}\left(\beta_{1}^{*}+\gamma_{1}^{\prime \prime} \cdot r_{20}^{\alpha_{1}}\right)^{1+\alpha_{2}}=\alpha_{1} \alpha_{2} \beta_{1}^{* \prime} \hat{\beta}_{2^{*}}
$$

Similarly,

$$
\begin{gathered}
\gamma_{1^{*}} \geq g\left(r_{1}\right):=r_{1}-\hat{\beta}_{1^{*}} \cdot \frac{r_{1}^{\alpha_{1} \alpha_{2}}}{\left(\beta_{2}^{*}+\gamma_{2}^{*} \cdot r_{1}^{\alpha_{2}}\right)^{\alpha_{1}}} . \\
g\left(r_{10}\right)=\min _{r_{1} \in(0,+\infty)} g\left(r_{1}\right), \text { and } \\
r_{10}^{1-\alpha_{1} \alpha_{2}}\left(\beta_{2}^{*}+\gamma_{2}^{*} \cdot r_{10}^{\alpha_{2}}\right)^{1+\alpha_{1}}=\alpha_{1} \alpha_{2} \beta_{2}^{*} \hat{\beta}_{1^{*}} .
\end{gathered}
$$

Taking $r_{1}=r_{10}$ and $r_{2}=r_{20}$, then the first inequality in (15) and (16) hold if $\gamma_{1^{*}} \geq g\left(r_{10}\right)$, $\gamma_{2^{*}} \geq g\left(r_{20}\right)$, which are just condition (13) and (14). The second inequalities hold directly by the choice of $R_{1}$ and $R_{2}$, and it would remain to prove that $r_{10}<R_{1}$ and $r_{20}<R_{2}$. This is easily verified through elementary computations.

$$
\begin{aligned}
R_{1} & =\frac{\beta_{1}^{*}}{r_{20}^{\alpha_{1}}+\gamma_{1}^{*}} \\
& =\frac{\beta_{1}^{*}+\gamma_{1}^{*} \cdot r_{20}^{\alpha_{1}}}{r_{20}^{\alpha_{1}}}
\end{aligned}
$$




$$
\begin{aligned}
& =\frac{\left(\alpha_{1} \alpha_{2} \beta_{1}^{*} \hat{\beta}_{2^{*}}\right)^{\frac{1}{1+\alpha_{2}}} \cdot r_{20}^{\frac{\alpha_{1} \alpha_{2}-1}{1+\alpha_{2}}}}{r_{20}^{\alpha_{1}}} \\
& =\left(\alpha_{1} \alpha_{2} \beta_{1}^{*} \hat{\beta}_{2^{*}}\right)^{\frac{1}{1+\alpha_{2}}} \cdot r_{20}^{-\frac{1+\alpha_{1}}{1+\alpha_{2}}} .
\end{aligned}
$$

The proof is the same as that in $R_{1}, R_{2}=\left(\alpha_{1} \alpha_{2} \beta_{2}^{\prime \prime} \hat{\beta}_{1^{*}}\right)^{\frac{1}{1+\alpha_{1}}} \cdot r_{10}^{-\frac{1+\alpha_{2}}{1+\alpha_{1}}}$.

Next, we will prove $r_{10}<R_{1}, r_{20}<R_{2}$, or equivalently,

$$
\begin{aligned}
& r_{10} r_{20}^{\frac{1+\alpha_{1}}{r_{2}}}<\left(\alpha_{1} \alpha_{2} \beta_{1}^{*} \hat{\beta}_{2^{*}}\right)^{\frac{1}{1+\alpha_{2}}}, \\
& r_{20} r_{10}^{1+\alpha_{2}}<\left(\alpha_{1} \alpha_{2} \beta_{2}^{*} \hat{\beta}_{1^{*}}\right)^{\frac{1}{1+\alpha_{1}}} .
\end{aligned}
$$

Namely,

$$
r_{10}^{1+\alpha_{2}} r_{20}^{1+\alpha_{1}}<\alpha_{1} \alpha_{2} \beta_{1}^{*} \hat{\beta}_{2^{*}}, r_{20}^{1+\alpha_{1}} r_{10}^{1+\alpha_{2}}<\alpha_{1} \alpha_{2} \beta_{2}^{*} \hat{\beta}_{1^{*}}
$$

On the other hand,

$$
r_{20}^{1-\alpha_{1} \alpha_{2}}\left(\beta_{1}^{*}\right)^{1+\alpha_{2}} \leq \alpha_{1} \alpha_{2} \beta_{1}^{*} \hat{\beta}_{2^{*}} .
$$

Then

$$
r_{20} \leq\left(\alpha_{1} \alpha_{2}\left(\beta_{1}^{*}\right)^{-\alpha_{2}} \hat{\beta}_{2^{*}}\right)^{\frac{1}{1-\alpha_{1} \alpha_{2}}} .
$$

Similarly

$$
r_{10} \leq\left(\alpha_{1} \alpha_{2}\left(\beta_{2}^{*}\right)^{-\alpha_{1}} \hat{\beta}_{1^{*}}\right)^{\frac{1}{1-\alpha_{1} \alpha_{2}}} .
$$

By (13) and (14),

$$
r_{10}^{1+\alpha_{2}} r_{20}^{1+\alpha_{1}} \leq\left(\alpha_{1} \alpha_{2}\left(\beta_{2}^{*}\right)^{-\alpha_{1}} \hat{\beta}_{1^{*}}\right)^{\frac{1+\alpha_{2}}{1-\alpha_{1} \alpha_{2}}}\left(\alpha_{1} \alpha_{2}\left(\beta_{1}^{*}\right)^{-\alpha_{2}} \hat{\beta}_{2^{*}}\right)^{\frac{1+\alpha_{1}}{1-\alpha_{1} \alpha_{2}}} .
$$

Now if we can prove

$$
\left(\alpha_{1} \alpha_{2}\left(\beta_{2}^{*}\right)^{-\alpha_{1}} \hat{\beta}_{1^{*}}\right)^{\frac{1+\alpha_{2}}{1-\alpha_{1} \alpha_{2}}}\left(\alpha_{1} \alpha_{2}\left(\beta_{1}^{*}\right)^{-\alpha_{2}} \hat{\beta}_{2^{*}}\right)^{\frac{1+\alpha_{1}}{1-\alpha_{1} \alpha_{2}}}<\alpha_{1} \alpha_{2} \beta_{1}^{*} \hat{\beta}_{2^{*}}
$$

then

$$
r_{10}^{1+\alpha_{2}} r_{20}^{1+\alpha_{1}}<\alpha_{1} \alpha_{2} \beta_{1}^{*} \hat{\beta}_{2^{*}}
$$

In fact,

$$
\left(\alpha_{1} \alpha_{2}\right)^{\frac{2+\alpha_{2}+\alpha_{1}-1}{1-\alpha_{1} \alpha_{2}}} \cdot\left(\frac{\hat{\beta}_{1^{*}}}{\beta_{1}^{*}}\right)^{\frac{1+\alpha_{2}}{1-\alpha_{1} \alpha_{2}}} \cdot\left(\frac{\hat{\beta}_{2^{*}}}{\beta_{2}^{*}}\right)^{\frac{\alpha_{1}\left(1+\alpha_{2}\right)}{1-\alpha_{1} \alpha_{2}}}<1,
$$

since $\hat{\beta} i^{*} \leq \beta_{i}^{*}, i=1,2$. Similarly,we have $r_{20}^{1+\alpha_{1}} r_{10}^{1+\alpha_{2}}<\alpha_{1} \alpha_{2} \beta_{2}^{*} \hat{\beta}_{1^{*}}$, we omit the details. Now we can obtain $r_{10}<R_{1}, r_{20}<R_{2}$. The proof is complete. 
The case $\gamma_{1}^{*} \leq 0, \gamma_{2^{*}}<0<\gamma_{2}^{*}\left(\gamma_{2}^{*} \leq 0, \gamma_{1^{*}}<0<\gamma_{1}^{*}\right)$

Theorem 6 Assume $(H)$ are satisfied. If $\gamma_{1}^{*} \leq 0, \gamma_{2^{*}}<0<\gamma_{2}^{*}$ and

$$
\begin{aligned}
& \gamma_{2^{*}} \geq\left(1-\frac{1}{\alpha_{1} \alpha_{2}}\right)\left[\alpha_{1} \alpha_{2} \frac{\hat{\beta}_{2^{*}}}{\left(\beta_{1}^{*}\right)^{\alpha_{2}}}\right]^{\frac{1}{1-\alpha_{1} \alpha_{2}}}, \\
& \gamma_{1^{*}} \geq r_{11}-\hat{\beta}_{1^{*}} \cdot \frac{r_{11}^{\alpha_{1} \alpha_{2}}}{\left(\beta_{2}^{*}+\gamma_{2}^{*} r_{11}^{\alpha_{2}}\right)^{\alpha_{1}}}
\end{aligned}
$$

where $0<r_{11}<+\infty$ is a unique positive solution of the equation

$$
r_{1}^{1-\alpha_{1} \alpha_{2}}\left(\beta_{2}^{* \prime}+\gamma_{2}^{\prime \prime} \cdot r_{1}^{\alpha_{2}}\right)^{1+\alpha_{1}}=\alpha_{1} \alpha_{2} \beta_{2}^{* \prime} \hat{\beta}_{1^{*}}
$$

then there exists a positive solution of (1).

Proof In this case,to prove that $A: K \rightarrow K$, it is sufficient to find $r_{1}<R_{1}, r_{2}<R_{2}$ such that

$$
\begin{array}{ll}
\frac{\hat{\beta}_{1^{*}}}{R_{2}^{\alpha_{1}}}+\gamma_{1^{*}} \geq r_{1}, & \frac{\beta_{1}^{*}}{r_{2}^{\alpha_{1}}} \leq R_{1}, \\
\frac{\hat{\beta}_{2^{*}}}{R_{1}^{\alpha_{2}}}+\gamma_{2^{*}} \geq r_{2}, & \frac{\beta_{2}^{*}}{r_{1}^{\alpha_{2}}}+\gamma_{2}^{*} \leq R_{2} .
\end{array}
$$

If we fix $R_{1}=\frac{\beta_{1}^{\prime \prime}}{r_{2}}, R_{2}=\frac{\beta_{2}^{\prime \prime}}{r_{1}^{\alpha_{2}}}+\gamma_{2}^{\prime \prime}$, then the first inequality of (21) holds if $r_{2}$ satisfies

$$
\gamma_{2^{*}} \geq r_{2}-\frac{\hat{\beta}_{2^{*}}}{R_{1}^{\alpha_{2}}}=r_{2}-\frac{\hat{\beta}_{2^{*}}}{\left(\beta_{1}^{*}\right)^{\alpha_{2}}} \cdot r_{2}^{\alpha_{1} \alpha_{2}}
$$

or equivalently

$$
\gamma_{2^{*}} \geq f\left(r_{2}\right):=r_{2}-\frac{\hat{\beta}_{2^{*}}}{\left(\beta_{1}^{*}\right)^{\alpha_{2}}} \cdot r_{2}^{\alpha_{1} \alpha_{2}}
$$

Then the function $f\left(r_{2}\right)$ possesses a minimum at

$$
r_{21}=\left[\alpha_{1} \alpha_{2} \cdot \frac{\hat{\beta}_{2^{*}}}{\left(\beta_{1}^{*}\right)^{\alpha_{2}}}\right]^{\frac{1}{1-\alpha_{1} \alpha_{2}}}
$$

i.e., $f\left(r_{21}\right)=\min _{r_{2} \epsilon(0,+\infty)} f\left(r_{2}\right)$.

On the analogy of (22), we obtain

$$
\gamma_{1^{*}} \geq r_{1}-\hat{\beta}_{1^{*}} \cdot \frac{r_{1}^{\alpha_{1} \alpha_{2}}}{\left(\beta_{2}^{*}+\gamma_{2}^{*} r_{1}^{\alpha_{2}}\right)^{\alpha_{1}}}
$$

or equivalently,

$$
\gamma_{1^{*}} \geq h\left(r_{1}\right):=r_{1}-\hat{\beta}_{1^{*}} \cdot \frac{r_{1}^{\alpha_{1} \alpha_{2}}}{\left(\beta_{2}^{*}+\gamma_{2}^{*} r_{1}^{\alpha_{2}}\right)^{\alpha_{1}}} .
$$


According to

$$
h^{\prime}\left(r_{1}\right):=1-\alpha_{1} \alpha_{2} \beta_{2}^{*} \hat{\beta}_{1^{*}} r_{1}^{\alpha_{1} \alpha_{2}-1}\left(\beta_{2}^{*}+\gamma_{2}^{*} r_{1}^{\alpha_{2}}\right)^{-1-\alpha_{1}}
$$

we have $h^{\prime}(0)=-\infty, h^{\prime}(+\infty)=1$, then there exists $r_{11}$ such that $h^{\prime}\left(r_{11}\right)=0$, and

$$
\begin{aligned}
h^{\prime \prime}\left(r_{1}\right)= & -\left[\alpha_{1} \alpha_{2} \beta_{2}^{*} \hat{\beta}_{1^{*}}\left(\alpha_{1} \alpha_{2}-1\right) r_{1}^{\alpha_{1} \alpha_{2}-2}\left(\beta_{2}^{*}+\gamma_{2}^{*} \cdot r_{1}^{\alpha_{2}}\right)^{-1-\alpha_{1}}\right. \\
& \left.+\alpha_{1} \alpha_{2} \beta_{2}^{*} \hat{\beta}_{1^{*}} r_{1}^{\alpha_{1} \alpha_{2}-1}\left(-1-\alpha_{1}\right)\left(\beta_{2}^{*}+\gamma_{2}^{*} \cdot r_{1}^{\alpha_{2}}\right)^{-2-\alpha_{1}} \gamma_{2}^{*} \alpha_{2} r_{1}^{\alpha_{2}-1}\right]>0 .
\end{aligned}
$$

Then the function $h\left(r_{1}\right)$ possesses a minimum at $r_{11}$, i.e., $h\left(r_{11}\right)=\min _{r_{1} \epsilon(0,+\infty)} f\left(r_{1}\right)$.

Note $h^{\prime}\left(r_{11}\right)=0$, then we have

$$
1-\alpha_{1} \alpha_{2} \beta_{2}^{*} \hat{\beta}_{1 *}^{*} r_{11}^{\alpha_{1} \alpha_{2}-1}\left(\beta_{2}^{*}+\gamma_{2}^{*} \cdot r_{11}^{\alpha_{2}}\right)^{-1-\alpha_{1}}=0
$$

Namely,

$$
r_{11}^{1-\alpha_{1} \alpha_{2}}\left(\beta_{2}^{*}+\gamma_{2}^{*} \cdot r_{11}^{\alpha_{2}}\right)^{1+\alpha_{1}}=\alpha_{1} \alpha_{2} \beta_{2}^{*} \hat{\beta}_{1^{*}}
$$

Taking $r_{2}=r_{21}$ and $r_{1}=r_{11}$ then the first inequality in (20) and (21) hold if $\gamma_{2^{*}} \geq h\left(r_{21}\right)$ and $\gamma_{1^{*}} \geq h\left(r_{11}\right)$ which are just condition (17) and (18). The second inequalities hold directly by the choice of $R_{2}$ and $R_{1}$, so it would remain to prove that $R_{1}=\frac{\beta_{1}^{*}}{r_{21}}>r_{11}, R_{2}=\frac{\beta_{2}^{*}}{r_{11}}+\gamma_{2}^{*}>r_{21}$. Now we turn to prove that $R_{1}>r_{11}, R_{2}>r_{21}$.

First,

$$
\begin{aligned}
& R_{1}=\frac{\beta_{1}^{*}}{r_{21}^{\alpha_{1}}}=\frac{\beta_{1}^{*}}{\left\{\left[\alpha_{1} \alpha_{2} \cdot \frac{\hat{\beta}_{2^{*}}^{*}}{\left(\beta_{1}^{*}\right)^{\alpha_{2}}}\right]^{\frac{1}{1-\alpha_{1} \alpha_{2}}}\right\}^{\alpha_{1}}} \\
& =\frac{\beta_{1}^{*}}{\left[\alpha_{1} \alpha_{2} \cdot \frac{\hat{\beta}_{2^{*}}}{\left(\beta_{1}^{*}\right)^{\alpha_{2}}}\right]^{\frac{\alpha_{1}}{1-\alpha_{1} \alpha_{2}}}}=\frac{\left(\beta_{1}^{*}\right)^{1+\frac{\alpha_{1} \alpha_{2}}{1-\alpha_{1} \alpha_{2}}}}{\left(\alpha_{1} \alpha_{2} \cdot \hat{\beta}_{2^{*}}\right)^{\frac{\alpha_{1}}{1-\alpha_{1} \alpha_{2}}}} \\
& =\frac{\left(\beta_{1}^{*}\right)^{\frac{1}{1-\alpha_{1} \alpha_{2}}}}{\left[\left(\alpha_{1} \alpha_{2} \cdot \hat{\beta}_{2^{*}}\right)^{\alpha_{1}}\right]^{\frac{1}{1-\alpha_{1} \alpha_{2}}}}=\left[\frac{\beta_{1}^{*}}{\left(\alpha_{1} \alpha_{2} \cdot \hat{\beta}_{2^{*}}\right)^{\alpha_{1}}}\right]^{\frac{1}{1-\alpha_{1} \alpha_{2}}} \\
& =\left[\frac{1}{\left(\alpha_{1} \alpha_{2}\right)^{\alpha_{1}}} \cdot \frac{\beta_{1}^{*}}{\left(\hat{\beta}_{2^{*}}\right)^{\alpha_{1}}}\right]^{\frac{1}{1-\alpha_{1} \alpha_{2}}}>\left[\alpha_{1} \alpha_{2} \cdot \frac{\hat{\beta}_{1^{*}}}{\left(\beta_{2}^{*}\right)^{\alpha_{1}}}\right]^{\frac{1}{1-\alpha_{1} \alpha_{2}}}=r_{11} \text {, }
\end{aligned}
$$

since $\hat{\beta}_{i^{*}} \leq \beta_{i}^{*}, i=1,2$.

On the other hand,

$$
R_{2}=\frac{\beta_{2}^{*}}{r_{11}^{\alpha_{2}}}+\gamma_{2}^{*}=\frac{\beta_{2}^{*}+\gamma_{2}^{*} \cdot r_{11}^{\alpha_{2}}}{r_{11}^{\alpha_{2}}} .
$$

By (18), we have

$$
\beta_{2}^{*}+\gamma_{2}^{*} \cdot r_{11}^{\alpha_{2}}=\left(\alpha_{1} \alpha_{2} \beta_{2}^{*} \hat{\beta}_{1^{*}}\right)^{\frac{1}{1+\alpha_{1}}} r_{11}^{\frac{\alpha_{1} \alpha_{2}-1}{1+\alpha_{1}}} .
$$


Combing (29) and (30),

$$
R_{2}=\left(\alpha_{1} \alpha_{2} \beta_{2}^{*} \hat{\beta}_{1^{*}}\right)^{\frac{1}{1+\alpha_{1}}} r_{11}^{-\frac{1+\alpha_{2}}{1+\alpha_{1}}}
$$

In what follows, we will verify that $R_{2}>r_{21}$. In fact,

$$
\left(\alpha_{1} \alpha_{2}\right)^{\frac{2+\alpha_{2}+\alpha_{1}}{1-\alpha_{1} \alpha_{2}}-1} \cdot\left(\frac{\hat{\beta}_{2^{*}}}{\beta_{2}^{*}}\right)^{\frac{1+\alpha_{1}}{1-\alpha_{1} \alpha_{2}}} \cdot\left(\frac{\hat{\beta}_{1^{*}}}{\beta_{1}^{*}}\right)^{\frac{\alpha_{2}\left(1+\alpha_{1}\right)}{1-\alpha_{1} \alpha_{2}}}<1
$$

since $\hat{\beta}_{i^{*}} \leq \beta_{i}^{*}, i=1,2$. Thus

$$
\left(\alpha_{1} \alpha_{2} \beta_{1}^{*\left(-\alpha_{2}\right)} \hat{\beta}_{2^{*}}\right)^{\frac{1+\alpha_{1}}{1-\alpha_{1} \alpha_{2}}} \cdot\left(\alpha_{1} \alpha_{2} \beta_{2}^{* \prime\left(-\alpha_{1}\right)} \hat{\beta}_{1^{*}}\right)^{\frac{1+\alpha_{2}}{1-\alpha_{2}}}<\alpha_{1} \alpha_{2} \beta_{2}^{*} \hat{\beta}_{1^{*}} \cdot
$$

On the other hand,

$$
\begin{aligned}
& r_{21}^{1-\alpha_{1} \alpha_{2}} \beta_{1}^{*\left(1+\alpha_{2}\right)} \leq \alpha_{1} \alpha_{2} \beta_{1}^{*} \hat{\beta}_{2^{*},}, \\
& r_{11}^{1-\alpha_{1} \alpha_{2}} \beta_{2}^{*\left(1+\alpha_{1}\right)} \leq \alpha_{1} \alpha_{2} \beta_{2}^{*} \hat{\beta}_{1^{*}} .
\end{aligned}
$$

Thus one can see easily note that

$$
\begin{aligned}
& r_{21} \leq\left(\alpha_{1} \alpha_{2} \beta_{1}^{*\left(-\alpha_{2}\right)} \hat{\beta}_{2^{*}}\right)^{\frac{1}{1-\alpha_{1} \alpha_{2}}}, \\
& r_{11} \leq\left(\alpha_{1} \alpha_{2} \beta_{2}^{*\left(-\alpha_{1}\right)} \hat{\beta}_{1^{*}}\right)^{\frac{1}{1-\alpha_{1} \alpha_{2}}} .
\end{aligned}
$$

From (33) and (34),

$$
r_{11}^{1+\alpha_{2}} r_{21}^{1+\alpha_{1}} \leq\left(\alpha_{1} \alpha_{2} \beta_{2}^{*\left(-\alpha_{1}\right)} \hat{\beta}_{1^{*}}\right)^{\frac{1+\alpha_{1}}{1-\alpha_{2}}}\left(\alpha_{1} \alpha_{2} \beta_{1}^{*\left(-\alpha_{2}\right)} \hat{\beta}_{2^{*}}\right)^{\frac{1+\alpha_{1}}{1-\alpha_{1} \alpha_{2}}} .
$$

Combing (32) and (35),

$$
r_{11}^{1+\alpha_{2}} r_{21}^{1+\alpha_{1}}<\alpha_{1} \alpha_{2} \beta_{2}^{*} \hat{\beta}_{1^{*}}
$$

Therefore,

$$
r_{21} r_{11}^{\frac{1+\alpha_{2}}{1+\alpha_{1}}}<\left(\alpha_{1} \alpha_{2} \beta_{2}^{*} \hat{\beta}_{1^{*}}\right)^{\frac{1}{1+\alpha_{1}}}
$$

Recall (31), we obtain $r_{21}<R_{2}$ immediately. The proof is complete.

Similarly, we have the following theorem.

Theorem 7 Assume (H) are satisfied. If $\gamma_{2}^{*} \leq 0, \gamma_{1^{*}}<0<\gamma_{1}^{*}$ and

$$
\begin{aligned}
& \gamma_{1^{*}} \geq\left(1-\frac{1}{\alpha_{1} \alpha_{2}}\right) \cdot\left[\alpha_{1} \alpha_{2} \frac{\hat{\beta}_{1^{*}}}{\left(\beta_{2}^{*}\right)^{\alpha_{1}}}\right]^{\frac{1}{1-\alpha_{1} \alpha_{2}}}, \\
& \gamma_{2^{*}} \geq r_{21}-\hat{\beta}_{2^{*}} \cdot \frac{r_{21}^{\alpha_{1} \alpha_{2}}}{\left(\beta_{1}^{*}+\gamma_{1}^{*} r_{21}^{\alpha_{1}}\right)^{\alpha_{2}}},
\end{aligned}
$$


where $0<r_{21}<+\infty$ is a unique positive solution of the equation

$$
r_{2}^{1-\alpha_{1} \alpha_{2}}\left(\beta_{1}^{*}+\gamma_{1}^{*} \cdot r_{2}^{\alpha_{1}}\right)^{1+\alpha_{2}}=\alpha_{1} \alpha_{2} \beta_{1}^{*} \hat{\beta}_{2^{*}}
$$

then there exists a positive solution of (1).

The case $\gamma_{1^{*}} \geq 0, \gamma_{2^{*}}<0<\gamma_{2}^{*}\left(\gamma_{2^{*}} \geq 0, \gamma_{1^{*}}<0<\gamma_{1}^{*}\right)$

Theorem 8 Assume $(H)$ are satisfied. If $\gamma_{1^{*}} \geq 0, \gamma_{2^{*}}<0<\gamma_{2}^{*}$ and

$$
\gamma_{2^{*}} \geq r_{22}-\hat{\beta}_{2^{*}} \cdot \frac{r_{22}^{\alpha_{1} \alpha_{2}}}{\left(\beta_{1}^{*}+\gamma_{1}^{*} r_{22}^{\alpha_{1}}\right)^{\alpha_{2}}}
$$

where $0<r_{22}<+\infty$ is a unique positive solution of the equation

$$
r_{2}^{1-\alpha_{1} \alpha_{2}}\left(\beta_{1}^{*}+\gamma_{1}^{*} \cdot r_{2}^{\alpha_{1}}\right)^{1+\alpha_{2}}=\alpha_{1} \alpha_{2} \beta_{1}^{*} \hat{\beta}_{2^{*}}
$$

then there exists a positive T-periodic solution of (1).

Proof In this case, to prove that $A: K \rightarrow K$, it is sufficient to find $r_{1}<R_{1}, r_{2}<R_{2}$ such that

$$
\begin{aligned}
& \frac{\hat{\beta}_{1^{*}}}{R_{2}^{\alpha_{1}}} \geq r_{1}, \quad \frac{\beta_{1}^{*}}{r_{2}^{\alpha_{1}}}+\gamma_{1}^{*} \leq R_{1} . \\
& \frac{\hat{\beta}_{2^{*}}}{R_{1}^{\alpha_{2}}}+\gamma_{2^{*}} \geq r_{2}, \quad \frac{\beta_{2}^{*}}{r_{1}^{\alpha_{2}}}+\gamma_{2}^{*} \leq R_{2} .
\end{aligned}
$$

If we fix $R_{1}=\frac{\beta_{1}^{*}}{r_{2}^{\alpha_{1}}}+\gamma_{1}^{*}, R_{2}=\frac{\beta_{2}^{*}}{r_{1}^{\alpha_{2}}}+\gamma_{2}^{*}$, then the first inequality of (37) satisfies

$$
\hat{\beta}_{2^{*}} \cdot\left(\frac{\beta_{1}^{*}}{r_{2}^{\alpha_{1}}}+\gamma_{1}^{*}\right)^{-\alpha_{2}}+\gamma_{2^{*}} \geq r_{2}
$$

or equivalently

$$
\gamma_{2^{*}} \geq l\left(r_{2}\right):=r_{2}-\frac{\hat{\beta}_{2^{*}}}{\left(\beta_{1}^{*}+\gamma_{1}^{*} r_{2}^{\alpha_{1}}\right)^{\alpha_{2}}} \cdot r_{2}^{\alpha_{1} \alpha_{2}}
$$

Then the function $l\left(r_{2}\right)$ possesses a minimum at $r_{22}$, i.e., $l\left(r_{22}\right)=\min _{r_{2} \epsilon(0,+\infty)} l\left(r_{2}\right)$.

Note $l^{\prime}\left(r_{22}\right)=0$, then we have

$$
1-\alpha_{1} \alpha_{2} \beta_{1}^{*} \hat{\beta}_{2^{*}} r_{22}^{\alpha_{1} \alpha_{2}-1}\left(\beta_{1}^{*}+\gamma_{1}^{*} \cdot r_{22}^{\alpha_{1}}\right)^{-1-\alpha_{2}}=0
$$

Therefore,

$$
r_{22}^{1-\alpha_{1} \alpha_{2}}\left(\beta_{1}^{*}+\gamma_{1}^{*} \cdot r_{22}^{\alpha_{1}}\right)^{1+\alpha_{2}}=\alpha_{1} \alpha_{2} \beta_{1}^{*} \hat{\beta}_{2^{*}}
$$

Note that $\hat{\beta} i^{*}, \beta_{i^{*}}>0, i=1,2$. And taking $r_{2}=r_{22}, R_{1}=\frac{\beta_{1}^{*}}{r_{22}}+\gamma_{1}^{*}, r_{1}=\frac{1}{R_{2}}$, it is sufficient to find $r_{1}<R_{1}, r_{2}<R_{2}$ such that

$$
R_{2}^{\alpha_{1}-1} \leq \beta_{1}^{*}, \quad R_{2}^{\alpha_{2}} \beta_{2}^{*}+\gamma_{2}^{*} \leq R_{2}
$$

and these inequalities hold for $R_{2}$ big enough because $\alpha_{i}<1$. The proof is completed. 
Similarly, we have the following theorem.

Theorem 9 Assume (H) are satisfied. If $\gamma_{2^{*}} \geq 0, \gamma_{1^{*}}<0<\gamma_{1}^{*}$ and

$$
\gamma_{1^{*}} \geq r_{12}-\hat{\beta}_{1^{*}} \cdot \frac{r_{12}^{\alpha_{1} \alpha_{2}}}{\left(\beta_{2}^{*}+\gamma_{2}^{*} r_{12}^{\alpha_{2}}\right)^{\alpha_{1}}},
$$

where $0<r_{12}<+\infty$ is a unique positive solution of the equation

$$
r_{1}^{1-\alpha_{1} \alpha_{2}}\left(\beta_{2}^{*}+\gamma_{2}^{*} \cdot r_{1}^{\alpha_{2}}\right)^{1+\alpha_{1}}=\alpha_{1} \alpha_{2} \beta_{2}^{*} \hat{\beta}_{1^{*}}
$$

then there exists a positive solution of (1).

\section{Competing interests}

The authors declare that they have no competing interests.

\section{Authors' contributions}

WL, YF, BS, and YP have critically revised the manuscript and have made substantial contributions to conception. WL designed the research and drafted the manuscript. YF, BS and YP revised it critically for important intellectual content. All authors read and approved the final manuscript.

\section{Author details}

'School of Management, Jilin University, Changchun, Jilin 130012, P.R. China. ${ }^{2}$ School of Mathematics, Jilin University, Changchun, Jilin 130012, P.R. China.

\section{Acknowledgement}

This study was supported by the National Science Founds of China (NO. 71172019).

Received: 7 May 2012 Accepted: 29 May 2012 Published: 2 July 2012

\section{References}

1. Agarwal, RP: Difference Equations and Inequalities: Theory, Methods and Applications. Dekker, New York (1992)

2. Agarwal, RP, Henderson, J: Positive solutions and nonlinear problems for third-order difference equation. Comput. Math. Appl. 36, 347-355 (1998)

3. Agarwal, RP, O'Regan, D: Multiple solutions for higher-order difference equations. Comput. Math. Appl. 37, 39-48 (1999)

4. Agarwal, RP, O'Regan, D: Discrete conjugate boundary value problems. Appl. Math. Lett. 37, 39-48 (1999)

5. Yuan, C: Positive solutions of a singular positone and semipositone boundary value problems for fourth-order difference equations. Discrete Dyn. Nat. Soc. 2010, Article ID 312864 (2010)

6. Avery, Rl, Chyan, CJ, Henderson, J: Twin solutions of boundary value problems for ordinary differential equations and finite difference equations. Comput. Math. Appl. 42, 695-704 (2001)

7. Eloe, PW: A generalization of concavity for finite differences. J. Math. Anal. Appl. 36(10-12), 109-113 (1998)

8. Erbe, LH, Xia, HX, Yu, JS: Global stability of linear non-autonomous delay difference equations. J. Differ. Equ. Appl. 1, 151-161 (1995)

9. Hao, ZC: Nonnegative solutions for semilinear third-order difference equation boundary value problems. Acta Sci. Math. 21A, 225-229 (2001) (in Chinese)

10. Henderson, J, Wong, PJY: Positive solutions for a system of nonpositive difference equations. Aequ. Math. 62, 249-261 (2001)

11. Kocic, VL, Ladas, G: Global Behavior of Nonlinear Difference Equations of Higher Order with Applications. Kluwer Academic, Boston (1993)

12. Hatsunaga, H, Hara, T, Sakata, S: Global attractivity for a nonlinear difference equation with variable delay. Comput. Math. Appl. 41, 543-551 (2001)

13. Zhang, B, Kong, L, Sun, Y, Deng, X: Existence of positive solutions for BVPs of fourth-order difference equations. Appl. Math. Comput. 131, 583-591 (2002)

14. Ma, R, Gao, C: Sign-changing solutions of nonlinear boundary value problems of difference equations. Indian J. Pure Appl. Math. 39, 323-332 (2008)

15. Bai, $\mathrm{D}, \mathrm{Xu}, \mathrm{Y}$ : Positive solutions for semipositone BVPs of second-order difference equations. Indian J. Pure Appl. Math. 39, 59-68 (2008)

16. $\mathrm{He}, \mathrm{Z}, \mathrm{Yu}, \mathrm{J}$ : On the existence of positive solutions of fourth-order difference equations. Appl. Math. Comput. 161, 139-148 (2005)

17. He, T, Su, Y: On discrete fourth-order boundary value problems with three parameters. J. Comput. Appl. Math. 233, 2506-2520 (2010)

18. Yuan, C, Jiang, D, O'Regan, D: Existence and uniqueness of positive solutions for fourth-order nonlinear singular continuous and discrete boundary value problems. Appl. Math. Comput. 203, 194-201 (2008) 
19. Jobe, J, Mingay, D: Cognition and survey measurement: history and overview. Appl. Cogn. Psychol. 5(3), 175-192 (1991)

20. Oppenheim, AN: Questionnaire Design, Interviewing and Attitude Measurement, 2nd edn. Continuum International Publishing, New York (1992)

21. Momani, SM: Some problems in non-Newtonian fluid mechanics. PhD thesis, Wales University, UK (1991)

22. Ma, TF, Silva, J: Iteration solution for a beam equation with nonlinear boundary conditions of third order. Appl. Math. Comput. 159, 11-18 (2004)

23. Chawla, MM, Katti, CP: Finite difference methods for two-point boundary-value problems involving higher order differential equation. BIT Numer. Math. 19, 27-33 (1979)

24. Pei, M, Chang, SK: Monotone iterative technique and symmetric positive solutions for a fourth-order boundary value problem. Math. Comput. Model. 51, 1260-1267 (2010)

25. Ma, D, Yang, X: Upper and lower solution method for fourth-order four-point boundary value problems. J. Comput. Appl. Math. 223, 543-551 (2009)

26. Feng, H, Ji, D, Ge, W: Existence and uniqueness of solutions for a fourth-order boundary value problem. Nonlinear Anal. 70, 3561-3566 (2009)

27. Ramadan, MA, Lashien, IF, Zahra, WK: Quintic nonpolynomial spline solutions for fourth-order two-point boundary value problem. Commun. Nonlinear Sci. Numer. Simul. 14, 1105-1114 (2009)

doi:10.1186/1687-1847-2012-97

Cite this article as: Li et al.: Positive solutions of a singular semipositone boundary value problems for fourth-order coupled difference equations. Advances in Difference Equations 2012 2012:97.

\section{Submit your manuscript to a SpringerOpen ${ }^{\circ}$ journal and benefit from:}

- Convenient online submission

- Rigorous peer review

- Immediate publication on acceptance

- Open access: articles freely available online

- High visibility within the field

- Retaining the copyright to your article 